$\begin{array}{cc}\text { Programa de Pós-Graduação em Engenharia de Produção - PPGEP } & \\ \text { Laboratório de Qualidade de Vida - LaQVida } & \text { REVISTA BRASILEIRA DE } \\ \text { Universidade Tecnológica Federal do Paraná - UTFPR } & \text { Ponta Grossa - PR - Brasil } \\ \text { v. 07, n. 01, jan./mar. 2015, p. } 48-55 & \text { QUALIDADE DE VIDA }\end{array}$

\title{
Relação entre o IMC e o IAC em meninos pré-escolares
}

\section{Relationship between BMI and BAI in pre school boys}

\author{
William Cordeiro de Souza \\ Universidade do Contestado - UnC - Porto União - Santa Catarina - Brasil \\ professor_williamsouza@yahoo.com.br \\ Marcos Tadeu Grzelczak \\ Universidade do Contestado - UnC - Porto União - Santa Catarina - Brasil \\ promarcostadeu@hotmail.com \\ Fernando Carvalheiro Reiser \\ Universidade de São Paulo -USP-São Paulo - São Paulo - Brasil \\ freiser@univali.br \\ Valderi Abreu de Lima \\ Universidade Federal do Paraná - UFPR - Curitiba - Paraná - Brasil \\ valderiabreulima@hotmail.com \\ Wallace Bruno de Souza \\ Universidade do Vale do Itajaí - Univali - Porto União - Santa Catarina - Brasil \\ wallace.bsouza@yahoo.com.br \\ Edgar Ismael Alarcón-Meza \\ Universidad Autónoma de Baja California - UABC - Mexicali - México \\ edva@uabc.edu.mx \\ Fabrício Faitarone Brasilino \\ Universidade da Região de Joinville - Univille - Joinville - Santa Catarina - Brasil \\ fabricio.brasilino@univille.net \\ Luis Paulo Gomes Mascarenhas \\ Universidade do Contestado - UnC - Porto União - Santa Catarina - Brasil \\ masca58@hotmail.com
}

\section{RESUMO}

OBJETIVO: O presente estudo buscou verificar a relação entre o Índice de Massa Corporal (IMC) e o Índice de Adiposidade Corporal (IAC) em meninos pré-escolares.

MÉTODOS: A amostra intencional foi constituída por pré-escolares $(n=73)$ do município de Três Barras - SC, do sexo masculino, com idade de 5,1 $\pm 0,1$ anos. Foram avaliados o peso e a estatura para cálculo do IMC. Para a classificação do IMC, utilizaram-se como referência as curvas de percentis do IMC para idade, conforme padrão de referência do Center for Disease Control (CDC) (2000). Para a determinação do IAC, foram coletados os dados da estatura e da circunferência quadril (CQ). Na determinação do IAC foi utilizada a equação convalidada para crianças proposta por El Aarbaoui et al. (2013), que sugere a fórmula: Índice de Adiposidade Corporal Pediátrica 
$(\mathrm{IACp})=$ Circunferência Quadril/Estatura ${ }^{0.8}$ - 38. Para a classificação do IAC utilizou-se como referência a tabela de classificação proposta por Bergman et al. (2011). Na análise dos dados foi utilizada a estatística descritiva - média e desvio padrão (dp) -, a frequência percentual (\%), a correlação de Pearson - (r) para verificar a relação entre os métodos do IMC e do IACp - e o teste de Bland Altman - para determinar o grau de concordância entre o IMC e o IAC. Recorreu-se ao teste do Qui-quadrado $\left(\mathrm{X}^{2}\right)$, para verificar a associação entre as frequências percentuais nos resultados obtidos entre os métodos utilizados, com nível de significância estipulado em $\mathrm{p}<0,05$.

RESULTADOS: Os resultados demonstraram uma correlação alta e significativa ( $\mathrm{r}=0,80$; $\mathrm{p}=<0,0001)$ entre as variáveis do IMC e do IACp. Foi possível verificar através do teste Quiquadrado, que não houve diferenças significativas entre as frequências percentuais nos resultados obtidos entre os dois métodos antropométricos.

CONCLUSÕES: Os resultados apresentados no presente estudo demonstram que o IACp é um método viável para diagnosticar a adiposidade corporal de crianças de cinco anos de idade em relação ao IMC.

PALAVRAS-CHAVE: Índice de massa corporal. Índice de adiposidade corporal. Sobrepeso. Obesidade. Escolares.

\section{ABSTRACT}

OBJECTIVE: This study looked at the relationship between BMI and BAI in boys pre-school.

METHODS: The sample was made up $(n=73)$ pre-school of Três Barras municipality - SC, male aged $5.1 \pm 0.1$ years. Weight and height was measured to obtain BMI calculated and used formula: $\mathrm{BMI}=$ Body Weight/height ${ }^{2}$. For the classification of BMI was used as reference percentile curves of BMI for age as the reference standard Center for Disease Control (CDC) (2000). To determine the BAI, we collected data of height and hip circumference (HC). In determining the BAI was used proposal equation for children proposed by El Aarbaoui et al. (2013) using the following formula: IACP $=$ Circumference Hip/Estatura ${ }^{0.8}$ - 38. For the classification of the BAI was used as reference table classification proposed by Bergman et al. (2011). The analysis of the data were used descriptive statistics - mean and standard deviation (SD) -, percentage rate (\%), the Pearson correlation was performed ( $r$ ) - to verify the relationship between anthropometric methods - and the Bland Altman method - for determination the degree of agreement between BMI and the BAI. Appealed to the chi-square (X2), to verify the association between the percentage frequencies in the results between the methods used, with significance level set at $\mathrm{p}<0.05$.

RESULTS: The results showed a high and significant correlation $(r=0.80, p=<0.0001)$ and BMI variables and the BAIp. It was verified by the chi-square test, no significant differences between the percentage frequencies in results between the two anthropometric methods.

CONCLUSIONS: The results presented in this study demonstrate that the BAIp is a viable method for diagnosing body fat of children under 5 years of age in relation to BMI.

KEYWORDS: Body mass index. Body adiposity index. Overweight. Obesity. School.

\section{Introdução}

O excesso de gordura corporal na infância está aumentando consideravelmente com o passar dos anos. Atualmente, no Brasil, o sobrepeso e a obesidade na faixa etária infantil, apresentaram um aumento significativo nas últimas décadas, sendo então considerados os desvios nutricionais mais relevantes para esta faixa etária (CONDE; MONTEIRO, 2006; SANT'ANNA et al., 2010).

Dessa forma, para auxiliar na identificação e na prevenção primária destas alterações, são necessários métodos para avaliar a composição corporal. Atualmente, existem vários indicadores 
antropométricos de obesidade total e central, dentre eles, destaca-se o Índice de Massa Corporal (IMC), o mais utilizado em estudos populacionais (SANT'ANNA et al., 2010).

O IMC é um dos indicadores antropométricos mais utilizados na avaliação do estado nutricional de populações e em estudos epidemiológicos. Entretanto, existem muitas limitações com relação ao seu uso, já que ele não é capaz de fornecer informações sobre a composição corporal e a distribuição da gordura corporal. A utilização desse método se torna bastante inconveniente principalmente quando a população que será avaliada apresenta um padrão de atividade física mais intenso. Pessoas com elevada quantidade de massa muscular podem apresentar elevado IMC mesmo que a gordura corporal não seja excessiva (REZENDE et al., 2007; GONÇALVES et al.; 2014).

Com bases nessas criticas, Bergman et al. (2011) realizaram uma pesquisa com AfricanoAmericanos tentando determinar a adiposidade com uma equação básica, chamada de Índice de Adiposidade Corporal (IAC), fácil de ser aplicada, com um gasto de tempo pequeno e que fosse mais precisamente aceitável que o próprio IMC. Utilizando-se das medidas de circunferência do quadril (CQ) e a altura, esse novo método pode ser utilizado para refletir o percentual de gordura corporal tanto para homens adultos quanto para mulheres de diferentes etnias, sem correção numérica. Para estruturação dessa nova fórmula foi usado o instrumento DEXA na avaliação do \%G, considerado padrão ouro. Encontrou-se uma forte correlação entre os parâmetros do IAC.

Depois do estudo de validação de Bergman et al. (2011), o IAC passou a ser visto como um bom e útil mecanismo para avaliação da saúde, podendo substituir outros IACs, incluindo o próprio IMC. Lópes et al. (2012) destacam em seu estudo que o IAC pode ser uma ferramenta tão fidedigna para medir a adiposidade quanto os equipamentos elétricos ou complexos sistemas mecânicos. Johnson et al. (2012) concluem seu estudo relatando que o IAC fornece um melhor indicador da adiposidade do que o IMC.

Com base nas controvérsias apresentadas, o presente estudo buscou como objetivo verificar a relação entre o IMC e o IAC em meninos pré-escolares do município de Três Barras - SC.

\section{Métodos}

A amostra intencional foi constituída por escolares $(n=73)$, do sexo masculino com idade de $5,1 \pm 0,1$ anos, todos pertencentes a uma escola da cidade de Três Barras, Santa Catarina. Os pais e os responsáveis pelos os alunos receberam um termo de consentimento para ser preenchido, no qual continha uma breve explicação dos objetivos e dos procedimentos metodológicos do estudo. Foram excluídas do estudo crianças que apresentaram doenças crônicas ou específicas do crescimento, que não compareceram na escola nos dias marcados para coleta de dados e as crianças cujos pais não autorizaram a participação. Sendo também respeitada a vontade das crianças que se recusaram a participar mesmo com a autorização dos pais. Esta pesquisa seguiu os princípios éticos de respeito à autonomia das pessoas, apontada pela Resolução no 196, de 10 de outubro de 1996 do Conselho Nacional de Saúde. É valido ressaltar que este trabalho foi aprovado pelo Comitê de Ética da Universidade do Contestado - UnC (número do Parecer: CAAE 05020512.7.0000.0117).

Foi avaliado o peso e a estatura para obtenção de IMC utilizando a seguinte formula: IMC= Peso Corporal/Estatura ${ }^{2}$. Na mensuração do peso sugerido por Fernandes Filho (2003), o avaliado deveria se posicionar em pé, de costas para escala da balança, usando o mínimo de roupa possível. A mensuração da estatura foi identificada pelo maior valor entre o vértex e a região plantar obedecendo ao plano de Frankfurt. A estatura foi verificada através de uma trena flexível marca Sanny Medical Sparrett, resolução de 0,1 mm, fixada na parede lisa, com 3 metros e graduação de $0,1 \mathrm{~cm}$ com o zero coincidindo com o solo.

Para determinação do peso corporal foi utilizada uma balança digital da marca Techline, devidamente calibrada, com graduação de 100 gramas e escalas variando de 0 a $180 \mathrm{Kg}$.

Para a classificação do IMC utilizou-se como referência as curvas de percentis do IMC, para idade, conforme padrão de referência do Center for Disease Control (CDC) (2000), atualmente recomendado pela Organização Mundial de Saúde que classifica: 
a) Baixo IMC valores <percentil 3;

b) IMC adequado ou eutrófico $>$ percentil 3 e $<$ percentil 85 ;

c) Sobrepeso > percentil 85 e < percentil 97;

d) Obesidade valores $>$ percentil 97.

Através desses dados foi classificado o sobrepeso e obesidade nas crianças.

Para a determinação do IAC, foram coletados os dados de estatura (já mencionado anteriormente) e a $\mathrm{CQ}$, que foi verificada através da extensão posterior máxima dos glúteos e foi tomada no nível dos pontos trocantéricos direito e esquerdo, realizada paralelamente ao solo, estando o avaliado com os pés unidos (HEYWARD; GIBSON, 2010). Para essa coleta foi utilizada uma trena antropométrica da marca Sanny Medical $2 \mathrm{mt}$ modelo SN-4011. Na determinação do IAC foi utilizada a equação convalidada para crianças proposta por El Aarbaoui et al. (2013) conhecida como Índice de Adiposidade Corporal Pediátrica (IACp), que utiliza à seguinte formula: IACp= Circunferência Quadril/Estatura ${ }^{0.8}$ - 38. Para a classificação do IAC utilizou-se como referência a tabela de classificação proposta por Bergman et al. (2011) que classifica o IAC:

a) Normal com o valor de 8 a 20;

b) Sobrepeso 21 a 25 ;

c) Obesidade acima de 25 .

Para a análise dos dados foi utilizada a estatística descritiva - média e desvio padrão (dp) -, a frequência percentual (\%), a correlação de Pearson (r) - para verificar a relação entre os métodos antropométricos - e o método de Bland Altman - para determinar o grau de concordância entre o IMC e o IAC. Recorreu-se ao teste do Qui-quadrado $\left(X^{2}\right)$, para verificar a associação entre as frequências percentuais nos resultados obtidos entre os métodos utilizados, com nível de significância estipulado em $\mathrm{p}<0,05$. Para o tratamento estatístico foi utilizado o software Med Calc.

\section{Resultados}

Na Tabela 1 estão apresentadas às características antropométricas dos meninos submetidos à avaliação para caracterização da amostra:

\begin{tabular}{ccc}
\multicolumn{3}{c}{ Tabela 1 - Características antropométricas dos avaliados } \\
\hline Variáveis & Média $(\mathrm{X})$ & Desvio Padrão (DP) \\
\hline Estatura $(\mathrm{m})$ & 108,0 & $\pm 0,05$ \\
Peso $(\mathrm{kg})$ & 19,0 & $\pm 3,41$ \\
IMC $\left(\mathrm{kg} / \mathrm{m}^{2}\right)$ & 15,81 & $\pm 1,76$ \\
Circ. do Quadril $(\mathrm{cm})$ & 60,0 & $\pm 4,50$ \\
IACp $\left(\mathrm{CQ} / \mathrm{m}^{0.8}-38\right)$ & 18,0 & $\pm 3,12$ \\
\hline
\end{tabular}

Fonte: Autoria própria (2014).

O Gráfico 1 está apresentando o diagrama de dispersão, demonstrando o valor da correlação encontrado, sendo que o mesmo apresenta pontos orientados em uma reta ascendente, caracterizando uma correlação alta e significativa $(\mathrm{r}=0,80 ; \mathrm{p}=<0,0001)$ entre as variáveis do IMC e do IACp.

Gráfico 1 - Diagrama de dispersão entre o IMC e o IACp

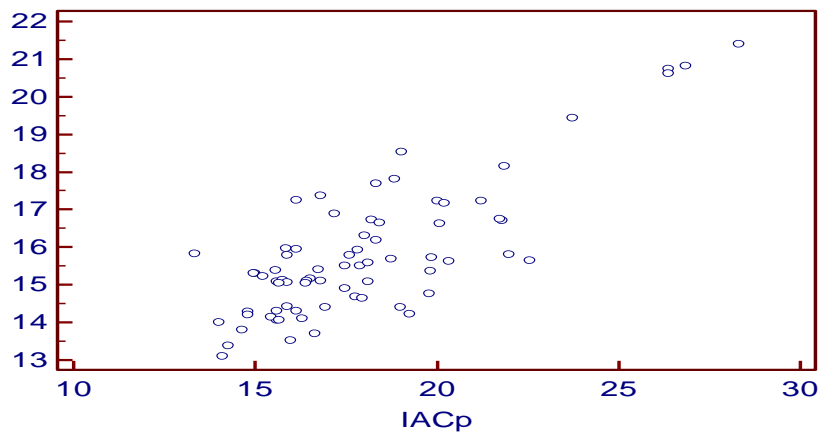

Fonte: Autoria própria (2014). 
O Gráfico 2 ilustra a concordância do IMC com IACp, através do método Bland-Altman. Esse método ajuda prover informações úteis no que diz respeito às faixas de valores para mostrar se as variáveis avaliadas são concordantes/discordantes (BERGMAN et al., 2011). Nesse estudo foi encontrado um intervalo de confiança concordante $(\mathrm{IC}=0,68$ a 0,86$)$.

Gráfico 2 - Concordância entre o IMC e o IACp utilizando método Bland-Altman

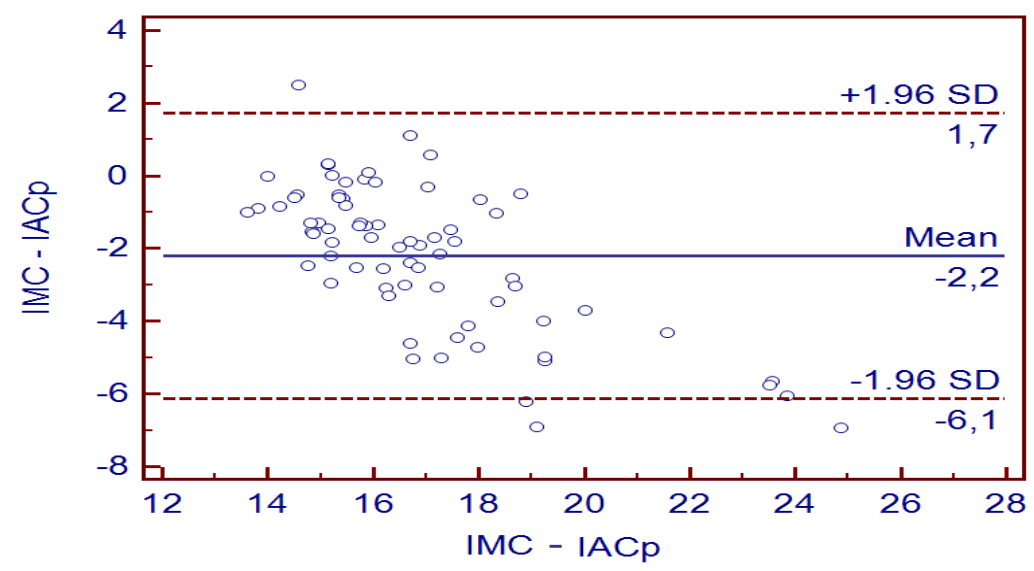

Fonte: Autoria própria (2014).

O Gráfico 3 apresenta as classificações encontradas nos métodos do IMC e do IAC, onde na classificação do IMC n=52 (71,23\%) dos avaliados estão classificadas como peso 'Normal Eutrófico', n=16 (21,92\%) com 'Sobrepeso' e n=5 (6,85\%) com 'Obesidade'.

Já no IACp n=66 (90,41\%) apresentaram sua adiposidade corporal como 'Eutrófico’ e n=7 $(9,59 \%)$ com sobrepeso. Foi possível verificar através do teste do Qui-quadrado, que não houve diferenças significativas entre as frequências percentuais nos resultados obtidos entre os métodos antropométricos.

Gráfico 3 - Percentagem das classificações dos métodos do IMC e IACp

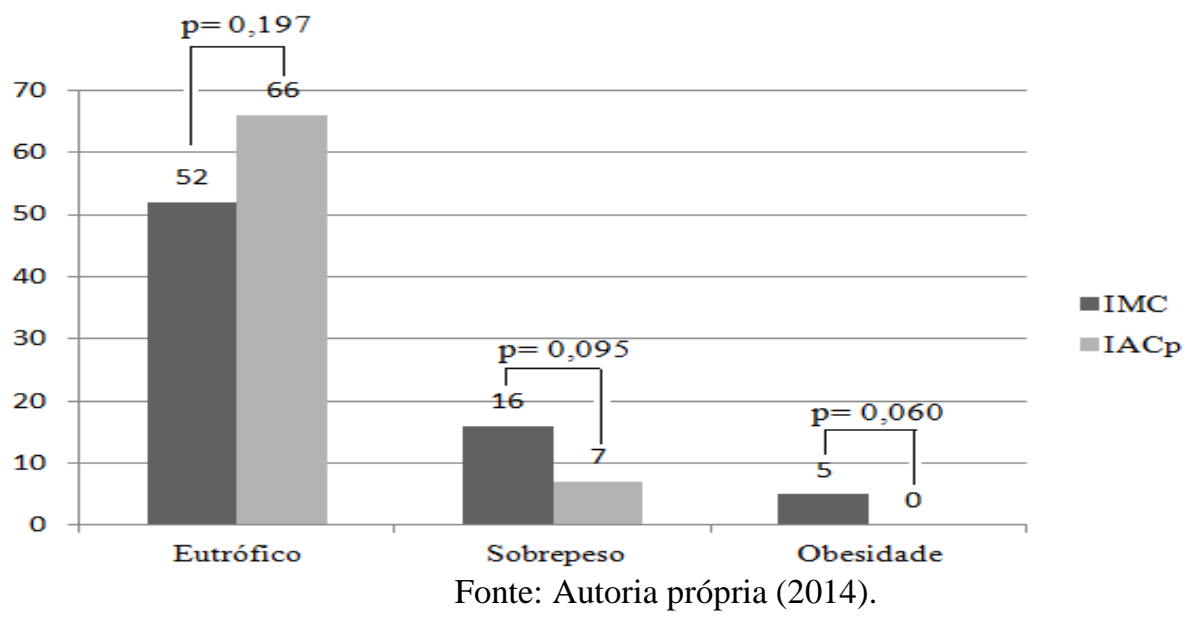

\section{Discussão}

Os resultados apresentados demonstraram uma correlação alta $(r=0,80 ; p=<0,0001)$ e um intervalo de confiança concordante (IC 95\%=0,68 a 0,86) entre as variáveis do IMC e do IACp. Os resultados obtidos nas classificações dos métodos do IMC e do IACp, através do teste Quiquadrado, mostraram resultados semelhantes, demonstrando que esses resultados não apresentaram diferenças significativas entre os métodos indiretos. Petroski (2011) destaca que, independente do método utilizado para determinar a composição corporal, é de suma importância monitorar as alterações do crescimento, do desenvolvimento e do nível de gordura. 
Entre os vários indicadores nutricionais a literatura destaca o índice de altura para a idade, uma vez que, este índice manifesta ser mais sensível e de extrema relevância para avaliar o processo de desnutrição ocorrido ao longo da vida da criança, pois reflete as condições de saúde e ambiental (MENEGOLLA et al., 2006). O peso também é indicador nutricional de boa precisão, pois fornece uma indicação de risco nutricional, termo preferencialmente utilizado, pois a desnutrição prenuncia um processo patológico basal em crianças (MOTTA et al., 2005).

Já a CQ se caracteriza como um indicador de gordura visceral e está diretamente associado ao risco de doenças como diabetes e doenças cardiovasculares tanto em crianças como em adultos (PETROSKI, 2011). Corroborando Yin, Lam e Janus (2003) destacam em seu estudo que tanto a estatura quando o peso corporal e a CQ apresentam forte associação aos fatores de risco cardiovascular, independente de faixa etária.

Petroski (2011) destaca que as equações antropométricas que utilizam as variáveis estatura, peso e circunferências são de extrema relevância, pois essas variáveis estão diretamente associadas a doenças, como por exemplo, as cardiovasculares.

Souza e Mascarenhas (2014) utilizaram a fórmula proposta por Bergman et al. (2011) com o objetivo de verificar o nível de aplicabilidade do IAC, comparado a outras técnicas indiretas de avaliação da composição corporal IMC e as dobras cutâneas. Os autores supracitados verificaram correlações significativas entre IMC e \%G ( $r=0,738$; $p<0,001)$, IMC e IAC ( $r=0,678$ e $p<0,001)$ e entre IAC e $\% \mathrm{G}(\mathrm{r}=0,687$ e $\mathrm{p}<0,001)$ em adolescentes do sexo masculino com idade entre 10 e 14 anos, concluindo que o IAC poderá ser um método viável para diagnosticar o estado nutricional. Dados estes que se assemelham ao presente estudo.

$\mathrm{Na}$ validação do IACp proposto por El Aarbaoui et al. (2013), em pesquisa realizada com 1.615 crianças com idades entre 5 a 12 anos a equação preditiva do IAC foi testada em sua correlação e concordância. Em seguida, a amostra foi dividida em duas sub-amostras para a construção de uma nova equação, a IACp. O IAC inicial superestimou o percentual de gordura corporal \%GC das crianças em 49\% (29,6 \pm 4,2\% versus 19,8 $\pm 6,8 \%$; $p<0,0001)$. Quando comparado o IAC com o IACp mostrou ter uma melhor correlação $(r=0,57 ; p<0,01$ versus $r=0,74$; $\mathrm{p}<0,0001$ ) e concordância ( $\mathrm{ICC}=0,34 ;[\mathrm{IC}=-0,19-0,65]$ versus $\mathrm{ICC}=0,83$; $[\mathrm{IC}=0,81-0,84]$ ). No entanto, houve alguns desvios sistemáticos entre os dois valores de \%GC como exemplificado pelo grande limite de $95 \%$ de concordância $[-9,1 \% ; 8,8 \%]$ obtido. O IAC superestima a \%GC em crianças. Em contraste, IACp aparece como um novo índice de gordura corporal para crianças, com precisão aceitável.

O estudo realizado por Romero-Velarde et al. (2014) tem o intuito de avaliar a associação do índice cintura-altura e do IAC com fatores de risco cardiovascular (FRCV) em crianças e adolescentes com obesidade. $\mathrm{O}$ índice cintura-altura mostrou correlação positiva e melhor do que o IAC com o percentual de gordura corporal por DEXA ( $\mathrm{r}=0,81$ vs 0,$75 ; \mathrm{p}<0,001)$ e de gordura abdominal ( $\mathrm{r}=0,79$ vs 0,$54 ; \mathrm{p}<0,001)$. Os valores médios de insulina e glicose, peso, estatura, CQ e IMC foram maiores em indivíduos com índice cintura-altura >0,6 ( $<<0,05)$; o colesterol HighDensity Lipoprotein HDL foi limítrofe $(\mathrm{p}=0,06)$. Os autores concluíram que em crianças e adolescentes com obesidade o índice cintura-altura é melhor do que o IAC para identificar indivíduos com adiposidade abdominal e fatores de risco cardiovascular; o ponto de corte de 0,6 de índice cintura-altura é adequado para identificar indivíduos com risco cardiovascular.

Contudo novas pesquisas precisam ser realizadas com a nova equação do IACp com o intuito de verificar sua associação com fatores de risco cardiovasculares, diabetes e outras patologias em crianças e em adolescentes.

\section{Considerações finais}

Os resultados do presente estudo demonstram que o IACp é um método viável para diagnosticar o estado nutricional de crianças de cinco anos de idade, aonde esse método apresentou um relação alta e significativa com o IMC, demonstrando ser um método fidedigno em medidas duplamente indiretas. 


\section{Referências}

BERGMAN, R. N.; STEFANOVSKI, D.; BUCHANAN, T. A.; SUMNER, A. E.; REYNOLDS, J. C.; SEBRING, G.; XIANG, A. H.; WATANABE, R. M. A better index of body adiposity. Obesity Journal, v. 19, n. 5, p. 1083-1089, 2011. rossef

CDC. Centers for disease control and prevention. National Center for Health Statistics. CDC Growth Charts. Atlanta: CDC, 2000. Disponível em: <http://www.cdc.gov/growthcharts>. Acesso em: 13 Feb. 2015.

CONDE, W. L.; MONTEIRO, C. A. Valores críticos do índice de massa corporal para classificação do estado nutricional de crianças e adolescentes brasileiros. Jornal de Pediatria, v. 82, p. 266-272, 2006. crossef

EL AARBAOUI, T.; SAMOUDA, H.; ZITOUNI, D.; DI POMPEO, C.; BEAUFORT, C.; TRICARETTO, F.; MORMENTYN, A.; HUBERT, H.; LEMDANI, M.; GUINHOUYA, B. C. Does the body adiposity index (BAI) apply to paediatric populations? Annals of Human Biology, v. 40, n. 5, p. 451-458, 2013. Crossef

FERNANDES FILHO, J. A prática da avaliação física: testes, medidas e avaliação física em escolares, atletas e academias de ginástica. 2. ed. Rio de Janeiro: Shape, 2003.

GONÇALVES, R.; MASCARENHAS, L. P. G.; LIEBL, E. C.; LIMA, V. A.; SOUZA, W. B.; GRZELCZAK, M. T.; SOUZA, W. C. Grau de concordância do IMC e do IAC com percentual de gordura. Revista Brasileira de Qualidade de Vida, v. 6, n. 1, p. 8-16, 2014. rossef

HEYWARD, V. H.; GIBSON, A. L. Advanced fitness assessment and exercise prescription. Champaign, IL: Human Kinetics, 2010.

JOHNSON, W.; CHUMLEA, W. C.; CZERWINSKI, S. A.; DEMERATH, E. W Concordance of the recently published body adiposity index with measured body fat percent in European-American adults. Obesity, v. 20, n. 4, p. 900-903, 2012. Crossef

LÓPEZ, A. A.; CESPEDES, M. L.; VICENTE, T.; TOMAS, M.; VENY, M. B.; TAULER, P.; AGUILO, A. Body adiposity index utilization in a Spanish Mediterranean population: comparison with the body mass index. PLoS ONE, v. 7, n. 4, p. 35281, 2012. crossef

MENEGOLLA, I. A.; DRACHLER, M. L.; RODRIGUES, I. H.; SCHWINGEL, L. R.; SCAPINELLO, E.; PEDROSO, M. B.; LEITE, J. C. C. Estado nutricional e fatores associados à estatura de crianças da Terra Indígena Guarita, Sul do Brasil. Caderno de Saúde Pública, v. 22, n. 2, p. 395-406, 2006. Crossef

MOTTA, M. E. F. A.; SILVA, G. A. P.; ARAÚJO, O. C.; LIRA, P. I.; LIMA, M. C. O peso ao nascer influencia o estado nutricional ao final do primeiro ano de vida?. Jornal de Pediatria, v. 85, n. 5, p. 377-382, 2005. Crossef

PETROSKI, E. L. Antropometria: técnicas e padronizações. 5. ed. São Paulo: Fontoura, 2011.

REZENDE, F.; ROSADO, L.; FRANCESCHINNI, S.; ROSADO, G.; RIBEIRO, R.; MARINS, J.

C. B. Revisão crítica dos métodos disponíveis para avaliar a composição corporal em grandes estudos populacionais e clínicos. Archivos Latinoamericanos de Nutrición, v. 57, n. 4, p. 327 334, 2007. 
ROMERO-VELARDE, E.; ALVAREZ-ROMAN, Y.; FONSECA-REYES, S.; STEIN, K.; TROYO-SANROMAN, R.; AGUIRRE-SALAS, L. Relationship between waist-height index and body adiposity index with cardiovascular risk factors in children and adolescents with obesity. The FASEB Journal, v. 28, n. 1, p. 641-645, 2014.

SANT’ANNA, M. S. L.; TINÔCO, A. L. A.; ROSADO, L. E. F. P. L.; SANT’ANA, L. F. R.; BRITO, I. S. S.; ARAÚJO, L. F.; MELLO, A. C.; SANTOS, T. F. Eficácia do índice de conicidade e da relação cintura/estatura em predizer o percentual de gordura corporal em crianças. Nutrire, v. 35, n. 2, p. 67-80, ago. 2010.

SOUZA, W. C.; MASCARENHAS, L. P. G. Aplicabilidade do índice de adiposidade corporal na estimativa da gordura corporal em escolares masculino. 2014. $10 \mathrm{f}$. Trabalho de Conclusão de Curso (Especialização em Fisiologia do Exercício com Ênfase em Treinamento) - Universidade São Fidélis, Rio de Janeiro, 2014.

YIN, S. H.; LAM, T. H.; JANUS, E. D. Waist to stature ratio is more strongly associated with cardiovascular risk factors than other simple anthropometric indices. Annals of Epidemiology, v. 13, n. 10, p. 683-691, 2003. Crossef 